\title{
Deuterium temperature, drift velocity, and density measurements in non-Maxwellian plasmas at ASDEX Upgrade
}

Salewski, M.; Geiger, B.; Jacobsen, A.S.; Abramovic, I.; Korsholm, S.B.; Leipold, F.; Madsen, B.; Madsen, J.; McDermott, R.M.; Moseev, D.

Total number of authors:

15

Published in:

Nuclear Fusion

Link to article, DOI:

10.1088/1741-4326/aaa6e1

Publication date:

2018

Document Version

Peer reviewed version

Link back to DTU Orbit

Citation (APA):

Salewski, M., Geiger, B., Jacobsen, A. S., Abramovic, I., Korsholm, S. B., Leipold, F., Madsen, B., Madsen, J., McDermott, R. M., Moseev, D., Nielsen, S. K., Nocente, M., Rasmussen, J., Stejner, M., \& Weiland, M. (2018). Deuterium temperature, drift velocity, and density measurements in non-Maxwellian plasmas at ASDEX Upgrade. Nuclear Fusion, 58(3), [036017]. https://doi.org/10.1088/1741-4326/aaa6e1

\section{General rights}

Copyright and moral rights for the publications made accessible in the public portal are retained by the authors and/or other copyright owners and it is a condition of accessing publications that users recognise and abide by the legal requirements associated with these rights.

- Users may download and print one copy of any publication from the public portal for the purpose of private study or research.

- You may not further distribute the material or use it for any profit-making activity or commercial gain

- You may freely distribute the URL identifying the publication in the public portal 
5 January 2018

\title{
Deuterium temperature, drift velocity, and density measurements in non-Maxwellian plasmas at ASDEX Upgrade
}

\author{
M Salewski ${ }^{1}$, B Geiger ${ }^{2}$, AS Jacobsen ${ }^{2}$, I Abramovic ${ }^{3}$, \\ SB Korsholm ${ }^{1}$, F Leipold ${ }^{1}$, B Madsen ${ }^{1}$, J Madsen ${ }^{1}$, \\ RM McDermott ${ }^{2}$, D Moseev ${ }^{3}$, SK Nielsen ${ }^{1}$, M Nocente ${ }^{4,5}$, \\ J Rasmussen ${ }^{1}$, M Stejner ${ }^{1}$, M Weiland ${ }^{2}$, the EUROfusion \\ MST1 team $\ddagger$ and the ASDEX Upgrade team $\S$ \\ 1 Technical University of Denmark, Department of Physics, Kgs. Lyngby, Denmark \\ ${ }^{4}$ University of Milano Bicocca, Department of Physics, Milano, Italy \\ ${ }^{5}$ Istituto di Fisica del Plasma, Consiglio Nazionale delle Ricerche, Milano, Italy \\ E-mail: msal@fysik.dtu.dk
}

2 Max-Planck-Institut für Plasmaphysik, Garching, Germany

3 Max-Planck-Institut für Plasmaphysik, Greifswald, Germany

\begin{abstract}
We measure the deuterium density, the parallel drift velocity, and parallel and perpendicular temperatures $\left(T_{\|}, T_{\perp}\right)$ in non-Maxwellian plasmas at ASDEX Upgrade. This is done by taking moments of the ion velocity distribution function measured by tomographic inversion of five simultaneously acquired spectra of $\mathrm{D}_{\alpha^{-}}$ light. Alternatively, we fit the spectra using a bi-Maxwellian distribution function. The measured kinetic temperatures $\left(T_{\|}=9 \mathrm{keV}, T_{\perp}=11 \mathrm{keV}\right)$ reveal the anisotropy of the plasma and are substantially higher than the measured boron temperature $(7 \mathrm{keV})$. The Maxwellian deuterium temperature computed with TRANSP $(6 \mathrm{keV})$ is not uniquely measurable due to the fast ions. Nevertheless, simulated kinetic temperatures accounting for fast ions based on TRANSP $\left(T_{\|}=8.3 \mathrm{keV}, T_{\perp}=10.4 \mathrm{keV}\right)$ are in excellent agreement with the measurements. Similarly, the Maxwellian deuterium drift velocity computed with TRANSP $(300 \mathrm{~km} / \mathrm{s})$ is not uniquely measurable, but the simulated kinetic drift velocity accounting for fast ions agrees with the measurements $(400 \mathrm{~km} / \mathrm{s})$ and is substantially larger than the measured boron drift velocity $(270 \mathrm{~km} / \mathrm{s})$. We further find that ion cyclotron resonance heating elevates $T_{\|}$ and $T_{\perp}$ each by $2 \mathrm{keV}$ without evidence for preferential heating in the $\mathrm{D}_{\alpha}$ spectra. Lastly, we derive an expression for the 1D projection of an arbitrarily drifting biMaxwellian onto a diagnostic line-of-sight.
\end{abstract}

$\ddagger$ See $[1]$

$\S$ See $[2]$ 


\section{Introduction}

Fusion plasmas are often described by just a few parameters summarizing their basic properties. The ion populations are described by the lowest moments of their velocity distribution functions: the density $n$, the drift velocity $v_{d}$ (or equivalently the so-called rotation), the temperature $T$, and the pressure $p$. Anisotropic plasmas are described by the temperatures $T_{\|}$and $T_{\perp}$ and the pressures $p_{\|}$and $p_{\perp}$ where the indices refer to directions with respect to the magnetic field.

However, the properties of the main-ion species are almost never measured directly, but are estimated from measurements of impurity ions and calculations. For example, deuterium temperatures and drift velocities are routinely estimated from the corresponding impurity parameters which are measured by charge-exchange recombination (CER) spectroscopy. A non-exhaustive list of examples of such measurements on a variety of tokamaks is found in references [3-23]. Deuterium temperatures and drift velocities are directly measured by $\mathrm{D}_{\alpha}$-based CER spectroscopy [24-34] and collective Thomson scattering (CTS) [35-44]. Neutron emission and gammaray spectroscopy also allow temperature measurements in high-performance plasmas [45]. A difficulty of main-ion measurements is that the associated distribution function is often highly non-Maxwellian and anisotropic due to the intense auxiliary plasma heating. The non-Maxwellian functional form and the anisotropy has up to now been dealt with by splitting the total population into a thermal, Maxwellian population and an energetic, non-Maxwellian population in analogy to the simulated populations in the widespread TRANSP code [46] (figure 1). In TRANSP fast ions are usually assumed to be part of the energetic population down to energies of $E=1.5 T_{i}$ where $T_{i}$ is the Maxwellian ion temperature. The fast ions with higher energies are tracked in the NUBEAM module [46], and when they have slowed down to an energy of $1.5 T_{i}$, they are removed from NUBEAM and added to the thermal population.

If the deviation from a Maxwellian is small, it is argued that the energetic-ion population is negligible [38-41]. In this case the total deuterium population is modelled as a thermal, Maxwellian population which is found as the best-fit Maxwellian to the total distribution function illustrated in figure 1(c). If the deviation from a Maxwellian is large, a common approach is to allow for the existence of an energetic, non-Maxwellian population in addition to a thermal population in analogy to TRANSP [28-31]. In this case one finds the best-fit Maxwellian to the distribution function illustrated in figure 1(a). However, as individual deuterium ions from the 'thermal' (figure 1(a)) and the 'energetic' (figure 1(b)) populations are indistinguishable, only the total main-ion

population (figure 1(c)) can be measured experimentally. We will show that the artificial splitting into thermal and non-thermal populations leads to ambiguity of the inferred bulk ion parameters. We will drop this artificial splitting altogether and consider one total deuterium ion population, and our goal will be to measure its lowest moments, the density, the drift velocity, and the parallel and perpendicular temperatures and pressures. 


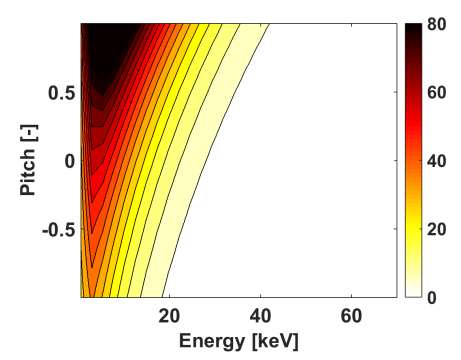

(a)

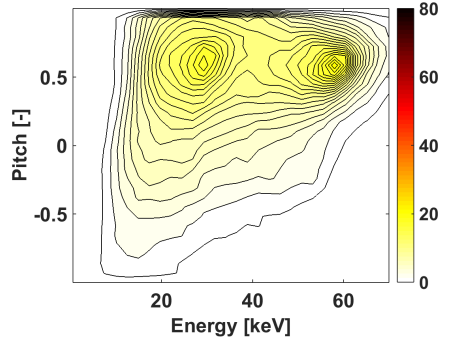

(b)

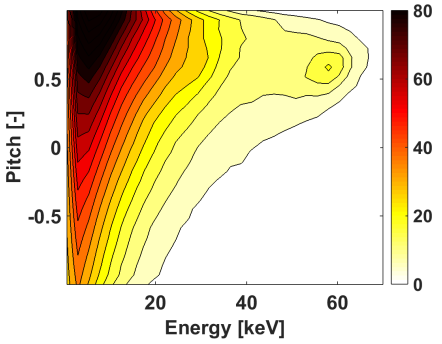

(c)

Figure 1. TRANSP splits the deuterium population into (a) a drifting Maxwellian and (b) a fast-ion velocity distribution function computed in the NUBEAM module in $\left[10^{16} /\left(\mathrm{keV} \mathrm{m}^{3}\right)\right]$. (c) The modelled complete deuterium velocity distribution is the sum of both populations. The parameters here are taken from a TRANSP simulation of ASDEX Upgrade [47] discharge \#32323 at $1711 \mathrm{~ms}$ in the plasma center just before a sawtooth crash. The NBI source is S3. The Maxwellian is described by $T=6 \mathrm{keV}$, $n_{t h}=2 \times 10^{19} \mathrm{~m}^{-3}$ and $v_{d}=3 \times 10^{5} \mathrm{~m} / \mathrm{s}$. The density of the fast-ion population in (b) is $n_{f}=6 \times 10^{18} \mathrm{~m}^{-3}$.

Here we demonstrate two new formalisms that accomplish this and account for the anisotropy and the deviation from a Maxwellian of the deuterium population. To this end, we have acquired five spectra of $\mathrm{D}_{\alpha}$-light originating from the same location simultaneously by active $\mathrm{D}_{\alpha}$-CER spectroscopy using five different lines-of-sight [48-50]. In our first approach we find the parallel-drifting bi-Maxwellian distribution function that produces the best fit to the five spectra. While this approach in principle allows measurements of the bi-Maxwellian parameters $n, v_{d \|}, T_{\|}, T_{\perp}, p_{\|}, p_{\perp}$ in plasmas that have a bi-Maxwellian distribution function, it is inaccurate for populations that are not Maxwellian or bi-Maxwellian as is often the case at ASDEX Upgrade. Nevertheless, this approach is still worth pursuing as it may be the only option for anisotropic temperature measurements if only two or three simultaneously acquired active CER measurements are available. Further, this approach has advantages compared with traditional CER spectroscopy based on one spectrum since measurements from several detectors are used simultaneously. This approach is hence a form of integrated data analysis [51].

In our second approach, we will not assume any functional form of the distribution function but instead measure the total deuterium distribution function by velocityspace tomography which is becoming an increasingly widespread tool to analyze fast-ion measurements [48-64]. We then calculate $n, v_{d \|}, T_{\|}, T_{\perp}, p_{\|}$and $p_{\perp}$ as the lowest moments of the total deuterium distribution function. These kinetic parameters are unique and well-defined for any distribution function in tokamak plasmas, and they reduce to the usual Maxwellian parameters if the distribution is Maxwellian.

This paper is organized as follows. Section 2 briefly reviews coordinate systems frequently used to measure drift velocities and ion distribution functions. We calculate the projection of a bi-Maxwellian distribution function onto a diagnostic line-of-sight in section 3, illustrating that perpendicular as well as parallel temperatures can 
be measured if spectroscopic data from two or more intersecting lines-of-sight are available. In section 4 we discuss measurements of the kinetic parallel and perpendicular temperatures and pressures, drift velocities and densities as moments of the velocity distribution function using velocity-space tomography. Section 5 gives an overview of the two discharges that we analyze. Sections 6 and 7 discuss difficulties one encounters when trying to fit non-Maxwellian distribution functions with Maxwellians and bi-Maxwellians in $1 \mathrm{D}$ and 2D, respectively. Section 8 presents measurement results obtained by fitting spectra with bi-Maxwellians and by velocity-space tomography. Finally, in section 9 conclusions are drawn.

\section{Bi-Maxwellians and drift velocities in common coordinate systems}

In this section we briefly define the various coordinate systems that are customary in descriptions of distribution functions and drift velocities in a tokamak. The drift velocities are often split into components in the toroidal and poloidal directions referring to a drift parallel to the magnetic axis and a drift azimuthally around it, respectively. As we analyze measurements in the plasma center in a high-power, high-torque plasma, the poloidal drift is not important compared with the toroidal drift. Often these drift velocities are presented in terms of so-called toroidal and poloidal rotations in units of $[\mathrm{Hz}]$ or $[\mathrm{rad} / \mathrm{s}]$. However, our formalism to calculate drift velocities and distribution functions is simplest in coordinates referring to parallel and perpendicular directions with respect to the local magnetic field vector due to the rotational symmetry associated with the rapid gyration of the ions. Vectors in these directions can easily be transformed to vectors in toroidal and poloidal directions since the local magnetic field vectors are known.

The anisotropic temperatures and pressures are most easily understood in $\left(v_{\|}, v_{\perp}\right)$ space which can be represented as a slice through the full $3 \mathrm{D}$ function $f_{v}^{3 D}\left(v_{\|}, v_{\perp}\right)$ with implied rotational symmetry or as a true $2 \mathrm{D}$ function $f_{v}^{2 D}\left(v_{\|}, v_{\perp}\right)$ with no implied third direction. These two functions are related by [52]

$$
f_{v}^{2 D}\left(v_{\|}, v_{\perp}\right)=2 \pi v_{\perp} f_{v}^{3 D}\left(v_{\|}, v_{\perp}\right)
$$

where the factor $v_{\perp}$ is the Jacobian of the transformation from Cartesian to cylindrical coordinates and $2 \pi$ is the integral over the ignorable gyroangle. The energy $E$ and pitch $\xi$ of a particle are another customary set of $2 \mathrm{D}$ coordinates. The $2 \mathrm{D}$ coordinate transformations between $f_{v}^{2 D}\left(v_{\|}, v_{\perp}\right)$ and $f(E, \xi)$ are

$$
\begin{array}{ll}
E=\frac{1}{2} m\left(v_{\|}^{2}+v_{\perp}^{2}\right) & v_{\|}=\xi \sqrt{\frac{2 E}{m}} \\
\xi=\frac{v_{\|}}{\sqrt{v_{\|}^{2}+v_{\perp}^{2}}} & v_{\perp}=\sqrt{1-\xi^{2}} \sqrt{\frac{2 E}{m}}
\end{array}
$$

with the Jacobians

$$
J_{v \rightarrow E, \xi}=\frac{1}{m \sqrt{1-\xi^{2}}} \quad J_{E, \xi \rightarrow v}=\frac{m v_{\perp}}{\sqrt{v_{\|}^{2}+v_{\perp}^{2}}} .
$$


A parallel-drifting bi-Maxwellian velocity distribution function in a magnetized plasma with the ignorable gyroangle $\gamma$ in $3 \mathrm{D}\left(v_{\|}, v_{\perp}\right)$-space is

$$
f_{v}^{3 D}\left(v_{\|}, v_{\perp}\right)=n\left(\frac{m}{2 \pi}\right)^{3 / 2} \frac{1}{T_{\perp} T_{\|}^{1 / 2}} \exp \left(-\frac{m\left(v_{\|}-v_{d \|}\right)^{2}}{2 T_{\|}}-\frac{m v_{\perp}^{2}}{2 T_{\perp}}\right) .
$$

A Maxwellian with perpendicular drift cannot be represented using the two coordinates $\left(v_{\|}, v_{\perp}\right)$ due to the rotational symmetry. A perpendicular drift velocity term of the form $\left(v_{\perp}-v_{d \perp}\right)^{2}$ would represent a ring distribution rather than a drifting Maxwellian due to the rotational symmetry. Therefore such a term is not included. It is possible to allow perpendicular drifts using the formalism shown in section 3 where one describes the rotation symmetric bi-Maxwellian in a coordinate system with a relative perpendicular velocity. In high-power, high-torque plasmas in ASDEX Upgrade, the perpendicular drift can be assumed to be small compared with the parallel drift in the plasma center where the pitch of the magnetic field lines is small. Therefore the 2D bi-Maxwellian is a good model that is often used. In $2 \mathrm{D}\left(v_{\|}, v_{\perp}\right)$-space it becomes according to equation 1

$$
f_{v}^{2 D}\left(v_{\|}, v_{\perp}\right)=n \frac{m^{3 / 2}}{(2 \pi)^{1 / 2}} \frac{v_{\perp}}{T_{\perp} T_{\|}^{1 / 2}} \exp \left(-\frac{m\left(v_{\|}-v_{d \|}\right)^{2}}{2 T_{\|}}-\frac{m v_{\perp}^{2}}{2 T_{\perp}}\right),
$$

and in 2D $(E, \xi)$-space according to equations 2 and 3

$$
f(E, \xi)=n\left(\frac{E}{\pi T_{\perp}^{2} T_{\|}}\right)^{1 / 2} \exp \left(-\frac{\xi^{2} E+\frac{1}{2} m v_{d \|}^{2}-v_{d \|} \xi \sqrt{2 m E}}{T_{\|}}-\frac{\left(1-\xi^{2}\right) E}{T_{\perp}}\right) .
$$

The standard drifting isotropic Maxwellians in these coordinate systems are obtained by setting $T_{\|}=T_{\perp}=T$ in equations 4 to 6 .

\section{Projection of a bi-Maxwellian with arbitrary drift}

For many diagnostics the projection of the distribution function onto a particular direction plays a special role. For example, the Doppler shift $\Delta \lambda$ in CER spectroscopy measurements is given by

$$
\Delta \lambda=\lambda_{0} \frac{u}{c}
$$

where $\lambda_{0}$ is the rest frame wavelength of the emitted light, $u$ is the velocity component along the line-of-sight and $c$ is the speed of light [65]. Blue- and red-shift correspond to negative and positive $u$, respectively. Similarly, the frequency shift of scattered radiation $\omega^{\delta}$ in CTS measurements is

$$
\omega^{\delta}=u\left|\mathbf{k}^{\delta}\right|
$$

where $\mathbf{k}^{\delta}$ is the difference between the wave vectors of received and incident radiation [66]. The velocity distribution function projected onto the line-of-sight for CER spectroscopy and onto $\mathbf{k}^{\delta}$ for CTS, $g(u)$, strongly influences the widths of the measured spectra. Here we derive an expression for the 1D projection of a bi-Maxwellian with arbitrary parallel and perpendicular drifts. This expression provides insight into 
temperature and drift velocity measurements in idealized bi-Maxwellian plasmas. The line-of-sight velocity component of a particle with velocities $\left(v_{\|}, v_{\perp}\right)$ and a perpendicular drift velocity $v_{d \perp}$ is

$$
u=v_{d \perp} \cos \beta+v_{\|} \cos \phi+v_{\perp} \sin \phi \cos \gamma
$$

where $\phi$ is the angle between the magnetic field and the line-of-sight and $\beta$ is the angle between the perpendicular drift velocity and the line-of-sight. Compared with the previously used projection equation [52], we here allow for a perpendicular drift velocity in analogy to the treatment of systematic Doppler shifts due to relative drifts of astrophysical rotating accretion discs to Earth [57]. $v_{\|}$is the parallel drift which is already handled by the existing formalism [52,67]. The projection of the 3D function onto the line-of-sight can be written using the Dirac $\delta$-function

$g(u, \phi)=\iiint f^{3 D}\left(v_{\|}, v_{\perp}\right) \delta\left(v_{\|} \cos \phi+v_{\perp} \sin \phi \cos \gamma-\left(u-v_{d \perp} \cos \beta\right)\right) v_{\perp} d v_{\|} d v_{\perp} d \gamma$

which can be interpreted as the projection onto the transformed coordinate

$$
u^{\prime}=u-v_{d \perp} \cos \beta .
$$

Integration over $\gamma$ gives for $\phi \neq 0[52]$

$$
g(u, \phi)=\int_{-\infty}^{\infty} \int_{\left(u^{\prime}-v_{\|} \cos \phi\right) / \sin \phi}^{\infty} \frac{2 f^{3 D}\left(v_{\|}, v_{\perp}\right)}{\sin \phi \sqrt{1-\left(\frac{u^{\prime}-v_{\|} \cos \phi}{v_{\perp} \sin \phi}\right)^{2}}} d v_{\perp} d v_{\|} .
$$

The lower integration limit $\left(u^{\prime}-v_{\|} \cos \phi\right) / \sin \phi$ in $v_{\perp}$ describes the border between the observable and unobservable velocity-space regions [52,67]. Unobservable regions can be identified here by a negative radicant in equation 12 . After substituting equation 4 and expanding the fraction by $v_{\perp}$, we get

$$
\begin{aligned}
g(u, \phi) & =\frac{2 n}{\sin \phi}\left(\frac{m}{2 \pi}\right)^{3 / 2} \frac{1}{T_{\perp} T_{\|}^{1 / 2}} \\
& \times \int_{-\infty}^{\infty} \int_{u^{\prime} / \sin \phi-v_{\|} \cot \phi}^{\infty} \frac{v_{\perp} \exp \left(-\frac{m v_{\perp}^{2}}{2 T_{\perp}}\right)}{\sqrt{v_{\perp}^{2}-\left(\frac{u^{\prime}-v_{\|} \cos \phi}{\sin \phi}\right)^{2}}} d v_{\perp} \exp \left(-\frac{m\left(v_{\|}-v_{d \|}\right)^{2}}{2 T_{\|}}\right) d v_{\|} .
\end{aligned}
$$

After integration over $v_{\perp}$,

$$
g(u, \phi)=\frac{n}{\sin \phi}\left(\frac{m}{2 \pi}\right) \frac{1}{\left(T_{\perp} T_{\|}\right)^{1 / 2}} \int_{-\infty}^{\infty} \exp \left(-\frac{m\left(\frac{u^{\prime}-v_{\|} \cos \phi}{\sin \phi}\right)^{2}}{2 T_{\perp}}-\frac{m\left(v_{\|}-v_{d \|}\right)^{2}}{2 T_{\|}}\right) d v_{\|},
$$

and over $v_{\|}$,

$$
g(u, \phi)=\frac{n}{\sin \phi}\left(\frac{m}{2 \pi}\right) \frac{1}{\left(T_{\perp} T_{\|}\right)^{1 / 2}}\left(\frac{2 \pi}{m\left(\frac{1}{T_{\|}}+\frac{1}{T_{\perp}} \cot ^{2} \phi\right)}\right)^{1 / 2}
$$




$$
\times \exp \left(-\frac{2 m^{2}\left(u^{\prime}-v_{d \|} \cos \phi\right)^{2}}{4 T_{\perp} T_{\|} m / 2\left(1 / T_{\perp}+1 / T_{\|}+\left(1 / T_{\perp}-1 / T_{\|}\right) \cos (2 \phi)\right)}\right),
$$

and after straightforward simplification and transformation back to $u$, we find an intuitive equation for the projection of the arbitrarily drifting bi-Maxwellian distribution function onto the line-of-sight:

$$
g(u, \phi)=n\left(\frac{m}{2 \pi\left(T_{\perp} \sin ^{2} \phi+T_{\|} \cos ^{2} \phi\right)}\right)^{1 / 2} \exp \left(-\frac{m\left(u-v_{d \|} \cos \phi-v_{d \perp} \cos \beta\right)^{2}}{2\left(T_{\|} \cos ^{2} \phi+T_{\perp} \sin ^{2} \phi\right)}\right) .
$$

Equation 16 is a 1D Maxwellian with the effective temperature in the $u$-coordinate along the line-of-sight

$$
T_{u}=T_{\perp} \sin ^{2} \phi+T_{\|} \cos ^{2} \phi
$$

and the $u$-drift

$$
u_{d}=v_{d \|} \cos \phi+v_{d \perp} \cos \beta .
$$

It connects the drifting 1D Maxwellian often used in temperature measurements to an underlying group of 2D arbitrarily drifting bi-Maxwellians with the same 1D projection. The angle between the line-of-sight and the magnetic field is always known. If the direction of the perpendicular drift velocity is known, we also know $\beta$ for each view. If at least two simultaneous measurements at different viewing angles on the same measurement volume are available and the perpendicular drift direction is known, we could find all parameters of a drifting bi-Maxwellian, assuming that the velocity distribution function has a bi-Maxwellian shape. This is accomplished by measuring $T_{u}$ and $u_{d}$ for each view (at least two views) and inverting equation 17 to obtain $T_{\|}$and $T_{\perp}$ and equation 18 to obtain $v_{d \|}$ and $v_{d \perp}$. We illustrate this possibility by considering the two extreme angles $\phi=0^{\circ}$ and $\phi=90^{\circ}$ leading to a particularly simple inversion. For $\phi=90^{\circ}$ the parallel temperature and the parallel drift velocity drop out. The width of the Maxwellian is then given by the perpendicular temperature and the drift is the projected perpendicular drift:

$$
g\left(u, \phi=90^{\circ}\right)=n\left(\frac{m}{2 \pi T_{\perp}}\right)^{1 / 2} \exp \left(-\frac{m\left(u-v_{d \perp} \cos \beta\right)^{2}}{2 T_{\perp}}\right) .
$$

For $\phi \rightarrow 0$ the perpendicular temperature and the perpendicular drift velocity drop out (as $\beta \rightarrow 90^{\circ}$ ). The width is given by the parallel temperature. The observed $u$-drift is $v_{d \|}$. The special case $\phi=0$ gives the same results as the limit $\phi \rightarrow 0$ of equation 16 (we omit the analogous derivation for brevity):

$$
g\left(u, \phi=0^{\circ}\right)=n\left(\frac{m}{2 \pi T_{\|}}\right)^{1 / 2} \exp \left(-\frac{m\left(u-v_{d \|}\right)^{2}}{2 T_{\|}}\right) .
$$

For $T_{\|}=T_{\perp} \equiv T, \phi$ drops out of the temperature terms, and we obtain a standard drifting Maxwellian with the same temperature for any $\phi$, but with $u$-drift velocities that do depend on the direction of the line-of-sight:

$$
g(u, \phi)=n\left(\frac{m}{2 \pi T}\right)^{1 / 2} \exp \left(-\frac{m\left(u-v_{d \|} \cos \phi-v_{d \perp} \cos \beta\right)^{2}}{2 T}\right) .
$$




\section{Kinetic temperatures, drift velocities and densities for arbitrary distribution functions}

This section provides definitions of the kinetic parallel and perpendicular temperatures, drift velocities and densities and briefly defines how to compute them from an arbitrary distribution function. At ASDEX Upgrade five active $\mathrm{D}_{\alpha}$-CER spectra are simultaneously measured using five different lines-of-sight [48]. These intersect the beam path of NBI source S3 in the same region in the plasma. Each line-of-sight forms a different angle with the local magnetic field vector, so that different parts of velocity space are observed [67]. For such a setup we can drop the assumption that the $1 \mathrm{D}$ projections of the distribution function are Maxwellian. Instead we can find the complete distribution function by velocity-space tomography which provides the best regularized fit to the measurement data. We can then summarize some of the rich information contained in the fitted distribution function by computing its lowest moments. In this section we briefly define these. The zeroth moment is the density:

$$
n=\int_{-\infty}^{\infty} f(\mathbf{v}) d \mathbf{v}=\int_{0}^{\infty} \int_{-\infty}^{\infty} f\left(v_{\|}, v_{\perp}\right) d v_{\|} d v_{\perp}=\int_{-1}^{1} \int_{0}^{\infty} f(E, \xi) d E d \xi .
$$

The first moment is the drift velocity:

$$
\mathbf{v}_{d}=\frac{1}{n} \int_{-\infty}^{\infty} \mathbf{v} f(\mathbf{v}) d \mathbf{v}
$$

The perpendicular drift is presently neglected in the tomographic inversion. The parallel drift velocity is

$$
v_{d \|}=\frac{1}{n} \int_{0}^{\infty} \int_{-\infty}^{\infty} v_{\|} f\left(v_{\|}, v_{\perp}\right) d v_{\|} d v_{\perp}=\frac{1}{n} \int_{-1}^{1} \int_{0}^{\infty} \xi \sqrt{\frac{2 E}{m}} f(E, \xi) d E d \xi .
$$

The second moment is known as the pressure tensor

$$
\mathbf{P}=m \int_{-\infty}^{\infty}\left(\mathbf{v}-\mathbf{v}_{d}\right)\left(\mathbf{v}-\mathbf{v}_{d}\right) f(\mathbf{v}) d \mathbf{v}
$$

which can, for rotational symmetry about the magnetic field, be written as

$$
\mathbf{P}=\left(\begin{array}{ccc}
p_{\perp} & 0 & 0 \\
0 & p_{\perp} & 0 \\
0 & 0 & p_{\|}
\end{array}\right)
$$

where the parallel and perpendicular kinetic pressures are

$$
\begin{aligned}
p_{\|} & =m \int_{0}^{\infty} \int_{-\infty}^{\infty}\left(v_{\|}-v_{d \|}\right)^{2} f\left(v_{\|}, v_{\perp}\right) d v_{\|} d v_{\perp} \\
& =m \int_{-1}^{1} \int_{0}^{\infty}\left(\xi \sqrt{\frac{2 E}{m}}-v_{d \|}\right)^{2} f(E, \xi) d E d \xi \\
p_{\perp} & =\frac{m}{2} \int_{0}^{\infty} \int_{-\infty}^{\infty} v_{\perp}^{2} f\left(v_{\|}, v_{\perp}\right) d v_{\|} d v_{\perp} \\
& =\int_{-1}^{1} \int_{0}^{\infty}\left(1-\xi^{2}\right) E f(E, \xi) d E d \xi .
\end{aligned}
$$


The total kinetic pressure is defined as one third of the trace of $\mathbf{P}$ :

$$
\begin{aligned}
p & =\frac{1}{3} \operatorname{tr}(\mathbf{P})=\frac{1}{3}\left(p_{\|}+2 p_{\perp}\right) \\
& =\frac{2}{3} \int_{0}^{\infty} \int_{-\infty}^{\infty}\left(\frac{1}{2} m\left(v_{\|}-v_{d \|}\right)^{2}+\frac{1}{2} m v_{\perp}^{2}\right) f\left(v_{\|}, v_{\perp}\right) d v_{\|} d v_{\perp} \\
& =\frac{2}{3} \int_{-1}^{1} \int_{0}^{\infty}\left(E-v_{d \|} \xi \sqrt{2 m E}+\frac{1}{2} m v_{d \|}^{2}\right) f(E, \xi) d E d \xi
\end{aligned}
$$

The corresponding kinetic temperatures are

$$
\begin{aligned}
T_{\|} & =\frac{p_{\|}}{n}, \\
T_{\perp} & =\frac{p_{\perp}}{n}, \\
T & =\frac{p}{n}=\frac{1}{3}\left(T_{\|}+2 T_{\perp}\right) .
\end{aligned}
$$

If the distribution is Maxwellian, these definitions for kinetic pressures and temperatures reduce to our thermodynamic notions.

\section{Overview of discharges \#32323 and \#33178}

In sections 6 and 7 we will investigate discharge \#32323 theoretically, and in section 8 we will present measurements in discharges \#32323 and \#33178. Here we give a brief overview of these discharges. Figure 2 presents time traces of the auxiliary heating power and the plasma stored energy $W_{M H D}$ as well as the impurity (boron) and electron temperatures in the plasma center and the line-integrated electron density. The auxiliary heating was by neutral beam injection (NBI) and electromagnetic wave heating in the electron cyclotron range of frequencies (ECRF) and in the ion cyclotron range of frequencies (ICRF). The measurement times are highlighted in grey. Discharge \#32323 has a very low density $\left(2 \times 10^{19} \mathrm{~m}^{-3}\right)$ and $2.5 \mathrm{MW}$ of NBI heating power (by NBI source S3) which leads to a high impurity temperature $(7 \mathrm{keV})$. We will show that the deuterium population in this discharge is non-Maxwellian and substantially hotter. Discharge \#33178 had a higher density $\left(\left(6-7 \times 10^{19} \mathrm{~m}^{-3}\right)\right)$ and $3.5 \mathrm{MW}$ NBI heating. We will compare the anisotropic deuterium temperatures with and without additional $4 \mathrm{MW}$ ICRF heating at $5.5 \mathrm{~s}$ and $7 \mathrm{~s}$, respectively.

Profiles of the temperatures and the toroidal drift velocities as function of the normalized toroidal magnetic flux $\rho_{t}$ for the three analyzed time points are presented in figure 3. The measured boron impurity temperatures and toroidal drift velocities are fairly close to corresponding neoclassical predictions for deuterium according to TRANSP in discharge \#33178 whereas they differ by up to about $10-15 \%$ in discharge \#32323. These neoclassical predictions assume Maxwellian distributions for deuterium and boron. In the next section we will show that such neoclassical predictions are not uniquely measurable quantities in non-Maxwellian plasmas due to the presence of fast ions. 

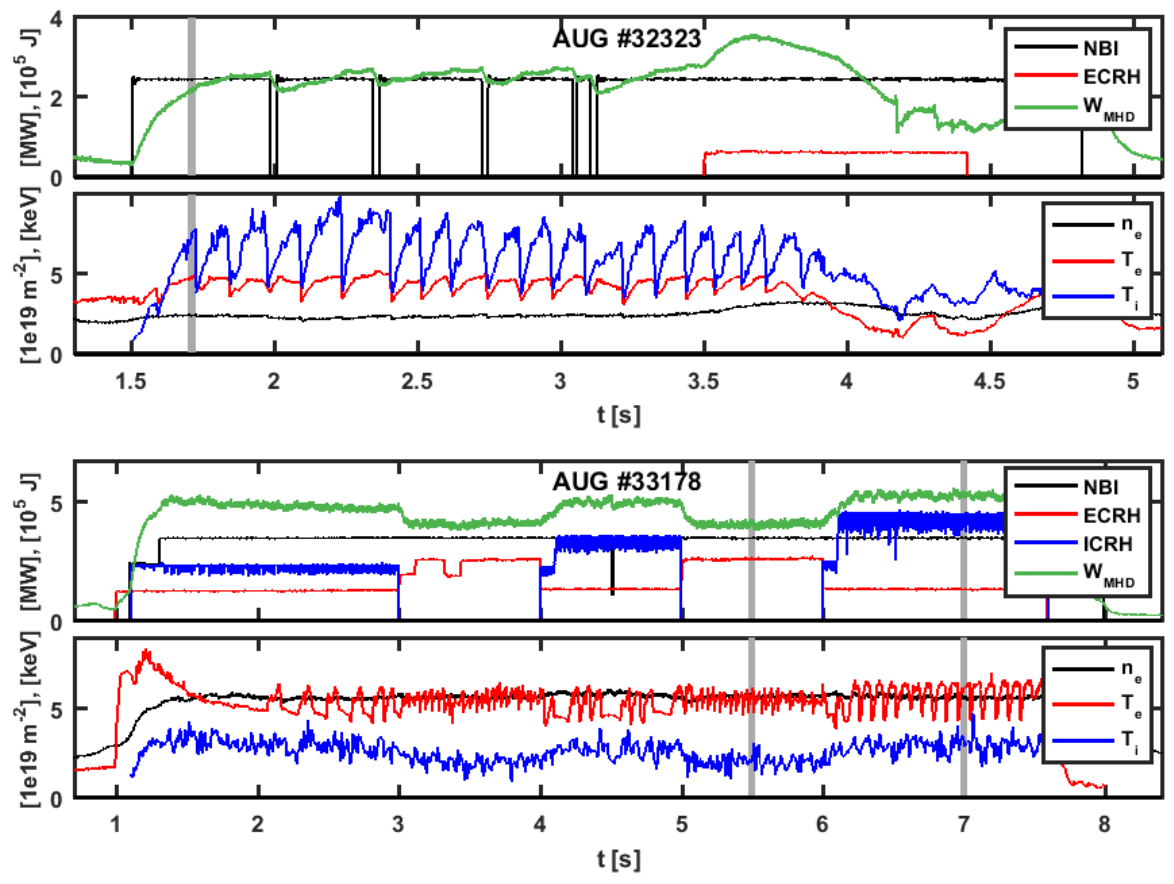

Figure 2. Overview of discharges \#32323 and \#33178. Time traces of NBI, ECRH and ICRH power and the plasma stored energy $W_{M H D}$ as well as the impurity and electron temperatures in the plasma center and the line-integrated electron density. The time points used in the analysis are highlighted in grey: $1.711 \mathrm{~s}$ in discharge \#32323 and $5.5 \mathrm{~s}$ and $7 \mathrm{~s}$ in discharge \#33178.

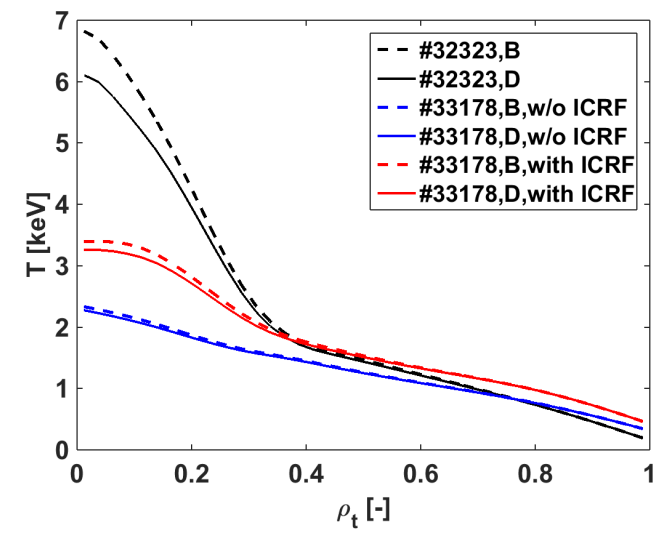

(a)

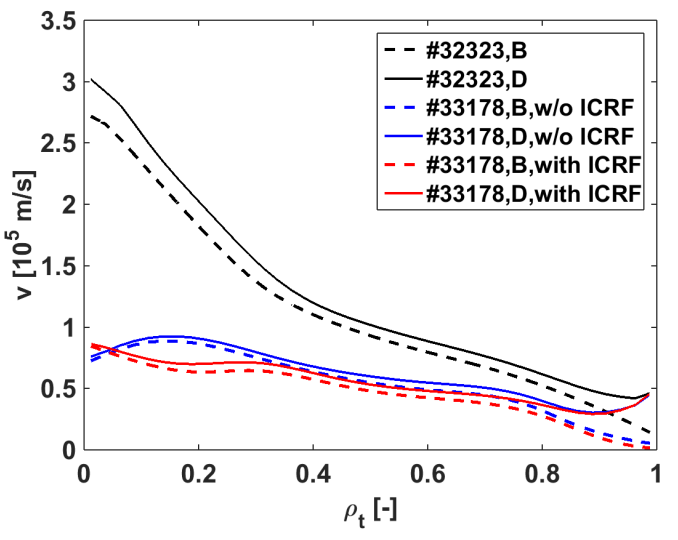

(b)

Figure 3. Profiles of measured boron (B) temperatures and toroidal drift velocities with corresponding neoclassical predictions for deuterium (D) according to TRANSP for the three measurements we will discuss. $\rho_{t}$ is the normalized toroidal magnetic flux. 


\section{1D fits of Maxwellians to non-Maxwellian distribution functions}

In this section we discuss difficulties in fitting 1D Maxwellians to non-Maxwellian deuterium populations as an underlying model for typical data analysis in $\mathrm{D}_{\alpha}$-based CER spectroscopy [24-34] and CTS [38-41]. Otherwise this section makes no reference to any particular diagnostic to gain insight into the basic difficulty. Whereas the Maxwellian velocity distribution function could be a good model for impurity species in the plasma, TRANSP simulations suggest that it can be a poor model for the main-ion species deuterium. The reason is that auxiliary heating selectively populates particular regions in velocity space. Deuterium ICRF and NBI heating therefore generate non-Maxwellian populations of deuterium ions.

Simulation codes such as the widely used TRANSP code deal with the nonMaxwellian ion population by artificially splitting the actual ion population into a thermal, Maxwellian ion population and an energetic $\left(E>1.5 T_{i}\right)$, non-Maxwellian population. In TRANSP the deuterium temperature, drift velocity and density of the introduced Maxwellian ion population are calculated based on other measurements, e.g. impurity temperatures and drift velocities. The fast-ion population is calculated by the NUBEAM module. The actual ion population is then modelled as the sum of the Maxwellian and the fast-ion population (figure 1).

However, the splitting of the total deuterium population into two artificial parts based on experimental data is ambiguous. Figure 4 illustrates this dilemma in a 1D example. We project the $2 \mathrm{D}$ velocity distribution functions from figure 1 onto a diagnostic line-of-sight at $\phi=80^{\circ}$ as discussed in section 3. The contributions from the Maxwellian population and the fast-ion population are plotted in blue as well as the total population (the sum) in black. However, in an experiment we can only measure the total population. The temperature of the Maxwellian according to TRANSP is $6 \mathrm{keV}$ which is the deuterium temperature neoclassically calculated from the impurity temperature. We also show an alternative Maxwellian at $7 \mathrm{keV}$ with the same density and its non-Maxwellian fast-ion complement summing up to the same total distribution. As there is no way to decide which splitting is best, the temperature, drift velocity and densities are ambiguous if a significant non-Maxwellian population is present. The same dilemma occurs for $2 \mathrm{D}$ velocity distribution functions.

An approach to bypass this dilemma is to assume that the energetic, nonMaxwellian population is negligible. Then the total deuterium population reduces to the Maxwellian part. In figure 5 we show temperatures and drift velocities found by fitting 1D Maxwellians to the total projected velocity distribution function from figure 4 . The $\mathrm{x}$-axis shows the interval in the projected velocity $u$ which is a proxy for the frequency or wavelength range of CTS or CER spectra used in the fit (equations 7 and 8). In CTS measurements, only measurement data at small projected velocities $u$ (or Doppler shifts) typical for thermal ions are used to measure temperatures [38-41]. However, figure 5 illustrates that the fitted temperature depends on the range of projected velocities used in the fit, in particular if this range is small. It appears to be best to use wide ranges so 


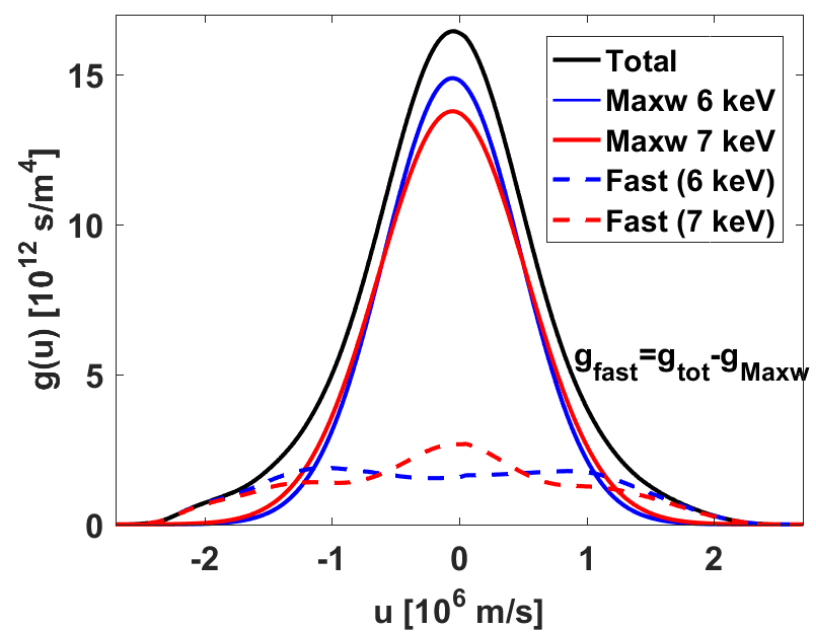

Figure 4. Projection of the velocity distribution functions simulated by TRANSP from figure 1 onto the line-of-sight $\left(\phi=80^{\circ}\right)$. The nominal temperature is $6 \mathrm{keV}$. Given only the total velocity distribution function (black), the splitting into thermal and fast-ion parts is not unique. This is illustrated by splitting the total distribution into two parts using Maxwellians with temperatures of $6 \mathrm{keV}$ and $7 \mathrm{keV}$ and their respective fast-ion distribution adding to the same total distribution.

that the fitted temperature does not strongly depend on the projected velocity range.

We further vary the adopted ratio of the densities of fast-ion and thermal populations, $n_{f} / n_{t h}$, which can be computed from the TRANSP simulation. The fitted temperatures and drift velocities depend on both parameters. The nominal density ratio according to TRANSP for the distributions shown in figure 1 is $n_{f} / n_{\text {th }}=0.3$. For ASDEX Upgrade discharge \#32323, the fitted temperature obtained from the total distribution according to TRANSP is about $9 \mathrm{keV}$ which is substantially higher than the nominal temperature according to TRANSP $(6 \mathrm{keV})$. The lower the density of fast ions, the more the fitted temperature to the total population approaches the nominal temperature.

Similar trends are observed for the drift velocity. Figure 5 suggests that we should expect to see differences between the fitted main-ion temperature and the corresponding nominal value from TRANSP due to the deviation from a Maxwellian. The density ratio $n_{f} / n_{t h}$ can be used to estimate how large this effect is for the discharge under consideration. This effect might partly explain the sometimes observed higher fitted temperatures and drift velocities of deuterium compared with the corresponding TRANSP estimates [41]. 


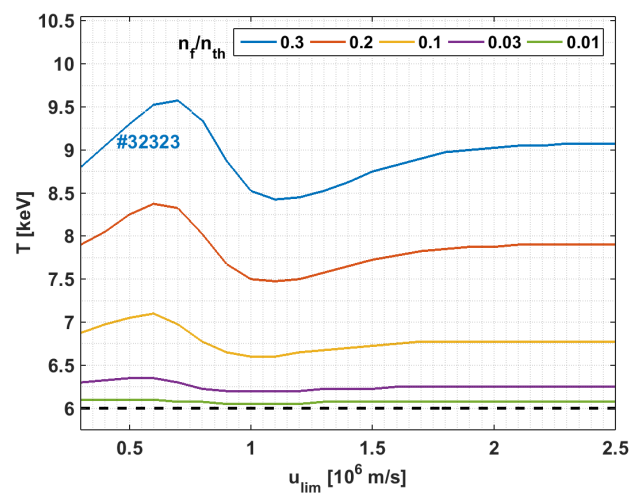

(a)

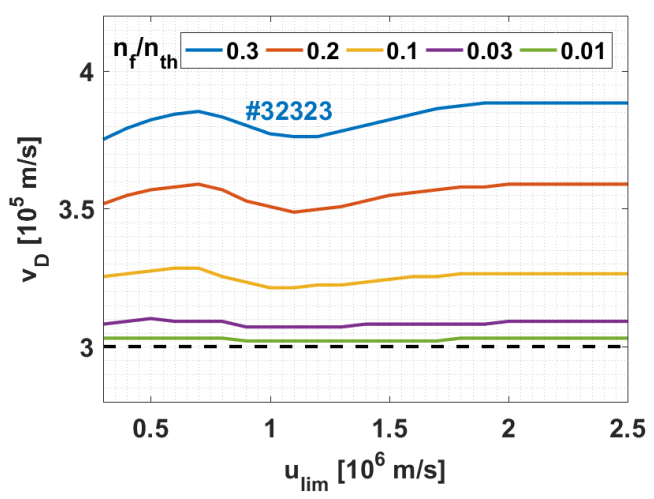

(b)

Figure 5. (a) Fitted temperatures $\left(\phi=80^{\circ}\right)$ and (b) parallel drift velocities $\left(\phi=10^{\circ}\right)$ assuming that the total velocity distribution function is Maxwellian. The $\mathrm{x}$-axes show the fitting interval in $u$ which is $\left[-u_{\text {lim }} ; u_{\text {lim }}\right]$ excluding the interval $[-2 ; 2] \times 10^{5} \mathrm{~m} / \mathrm{s}$ which is typically not experimentally accessible due to the need for a notch filter blocking e.g. the gyrotron radiation or the cold $\mathrm{D}_{\alpha}$-line. The angles $\phi$ are judiciously selected to illustrate large temperature and drift velocity changes.

\section{2D fits of Maxwellians and bi-Maxwellians to non-Maxwellian distribution functions}

The 1D examples hinted that the fitted temperatures and drift velocities depend on the fast ion population which is strongly non-Maxwellian. In figure 6 we consider an analogous $2 \mathrm{D}$ example, focusing just on the $2 \mathrm{D}$ velocity distribution function underlying the measurements but without reference to any particular diagnostic or line-of-sight. We consider again the TRANSP simulation illustrated in figure 1 where the total deuterium population is modelled to consist of a thermal, Maxwellian part and an energetic ion part computed by NUBEAM. Here we compute the kinetic drift velocities and temperatures of the total velocity distribution function according to section 4 and compare these with the corresponding parameters obtained by fitting bi-Maxwellians and Maxwellians to the total (non-Maxwellian) velocity distribution function. This modelled total distribution is illustrated in figure 1(c). Mathematically, we find the fitted parallel and perpendicular temperatures and parallel drift velocities by solving the minimization problem

$$
\operatorname{minimize}_{v_{d}, T_{\|}, T_{\perp}}\left(f_{\text {Maxw }}\left(E, \xi, n_{t o t}, v_{d}, T_{\|}, T_{\perp}\right)-f_{t o t}(E, \xi)\right)
$$

where $f_{\text {tot }}(E, \xi)$ is the total distribution in figure 1(c). This non-weighted minimization is done for the velocity-space up to $70 \mathrm{keV}$ as illustrated in figure 1 . The best-fitting single temperature Maxwellian is obtained with the same formalism and the contraint $T=T_{\|}=T_{\perp}$.

We again vary the ratio $n_{f} / n_{t h}$. In the case of bi-Maxwellian populations, the kinetic parameters are the same as the fitted bi-Maxwellian parameters. If $T_{\|}=T_{\perp}$, the bi-Maxwellian further reduces to the Maxwellian. This limit is approached for $n_{f} / n_{t h} \ll 1$ where all temperatures approach $6 \mathrm{keV}$ (figure 6(a)) and the parallel drift 
velocity $3 \times 10^{5} \mathrm{~m} / \mathrm{s}$ (figure $6(\mathrm{~b})$ ). For larger $n_{f} / n_{t h}$ the kinetic temperatures and the drift velocity increase due to the presence of fast ions. The perpendicular kinetic temperature is larger than the parallel kinetic temperature for NBI source S3 at ASDEX Upgrade due to the beam geometry (injection pitch $p \sim 0.6$ in the plasma center as illustrated in figure $1(\mathrm{~b})$ ). The total kinetic temperature is a 2:1 weighted average between the perpendicular and parallel kinetic temperatures (equation 32). Figure 6(b) shows an analogous comparison of fitted parallel drift velocities and the corresponding kinetic parallel drift velocities computed as moment of the total velocity distribution function. The fitted parallel drift velocities remain fairly constant for $n_{f} / n_{t h}<0.3$ and then increase strongly whereas the kinetic parallel drift velocity increases smoothly.

The bi-Maxwellian temperatures also increase with the ratio $n_{f} / n_{t h}$ but for $n_{f} / n_{t h}=0.3$, as in ASDEX Upgrade discharge \#32323, the fitted parallel temperature is larger than the fitted perpendicular temperature in disagreement with the corresponding kinetic temperatures. The difference to the corresponding kinetic values is about $2 \mathrm{keV}$. The differences in these temperatures and drift velocities suggest that the drifting Maxwellian and the drifting bi-Maxwellian models do not describe typical distribution functions in a low-density, NBI heated ASDEX Upgrade plasma well. The best fits have a too low drift velocity up to well beyond $n_{f} / n_{t h}=0.3$ which might be related to the too high parallel and too low perpendicular temperatures of the best fit.

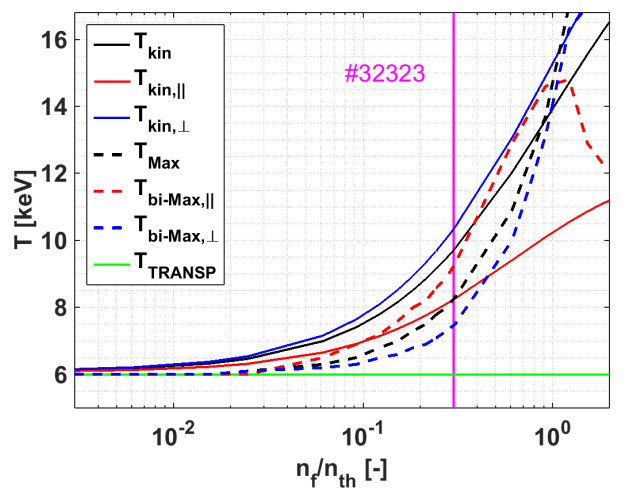

(a)

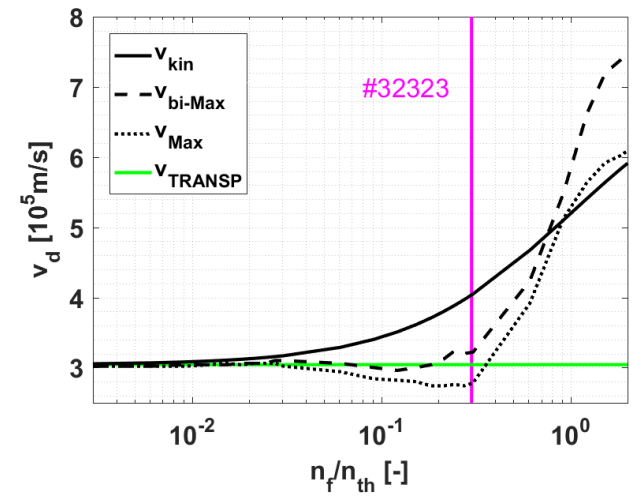

(b)

Figure 6. Kinetic temperatures and drift velocities as function of the adopted density ratio of the fast ions and thermal ions in the TRANSP picture. The nominal TRANSP deuterium values are $T=6 \mathrm{keV}$ and $v_{d}=300 \mathrm{~km} / \mathrm{s}$. Results from fits of Maxwellians and bi-Maxwellians to the total distribution are also shown. 


\section{Measurement results}

In previous sections we have studied how energetic ion populations due to auxiliary plasma heating influence measurements of main-ion temperatures based on theoretical considerations. It appeared that unique densities, drift velocities and anisotropic temperatures could be obtained by considering the total ion velocity distribution function, rather than an artificially introduced thermal part. In this section we develop two formalisms based on these findings allowing for possible anisotropy in the plasma, and we demonstrate them using experimental data. We infer temperatures following two new approaches exploiting that the five active $\mathrm{D}_{\alpha}$-based $\mathrm{CER}$ views allow measurements of kinetic anisotropy. Both approaches rely on the weight function formalism [52,67-72].

\subsection{Fits to the $D_{\alpha}$ spectra using bi-Maxwellians}

In previous applications of weight functions, the total ion distribution has been split into thermal and non-thermal parts. The weight functions depend only on thermal parameters in this picture, whereas they are applied only to energetic, non-thermal ions. Hence we could relate the measurable signal $S$ to the fast-ion velocity distribution function $F$ by the linear matrix equation

$$
S=W F
$$

where $W$ is a matrix composed of weight functions [53]. However, as we now seek to infer the total deuterium distribution function, the weight functions now depend on the function that we seek to infer, and we obtain a non-linear problem

$$
S=W(F) F .
$$

The weight functions $W(F)$ can be computed for arbitrary velocity distribution functions but this is computationally very demanding. Here we seek a computationally faster approach and generate a database of weight functions for bi-Maxwellian parameters. The best fitting bi-Maxwellian is found by solving the minimization problem

$$
\text { minimize }\left\|S-W\left(F_{b i M a x}\right) F_{b i M a x}\right\|_{2}
$$

where $W\left(F_{b i M a x}\right)$ is the weight function matrix computed using the bi-Maxwellian test functions. The weight functions have been precomputed for a discretization in the parameters $\left(n, v_{d}, T_{\|}, T_{\perp}\right)$. This allows us to find the best fitting drifting bi-Maxwellian to the five simultaneously measured spectra given the physics model encoded in the weight functions. Traditionally, one 1D Maxwellian is fit to one CER spectrum. Our approach combines measurements from various detectors allowing integrated data analysis. Furthermore, the weight function formalism accounts for the effect of the halo and the variable charge-exchange probabilities for different energies, pitches, and gyro-angles $[67,73]$. As consequence we do not obtain any apparent temperatures and apparent drift velocities that need to be corrected [74]. If the velocity distribution 
function is close to bi-Maxwellian, all parameters can be found rapidly by matrix multiplication.

We applied this formalism to the five simultaneously measured $\mathrm{D}_{\alpha}$-based CER spectra in ASDEX Upgrade discharge \#32323 obtained for the plasma described in figure 1 [50]. With this approach we find parallel and perpendicular temperatures that are consistently higher than the deuterium temperature of $6 \mathrm{keV}$ neoclassically determined by TRANSP based on the measured boron temperature. However, the values vary substantially in the range $7 \mathrm{keV}$ to about $13 \mathrm{keV}$ (depending on used data ranges) and either the parallel or perpendicular temperature of the fitted bi-Maxwellian is higher. This behavior is consistent with the difficulty of fitting a Maxwellian or bi-Maxwellian to a realistic total distribution function as these are not good models in this case (section 7). While this approach works very well using synthetic data and presumably also in bi-Maxwellian plasma, we do not obtain unique results in the strongly non-Maxwellian plasma in discharge \#32323 investigated here.

\subsection{Temperature and drift velocity measurements by velocity-space tomography}

Our second new approach to measure bulk plasma parameters is to find the best fitting smooth velocity distribution function by velocity-space tomography based on the five spectra of $\mathrm{D}_{\alpha}$-light. In this approach no specific functional form of the ion velocity distribution function is assumed, such as a Maxwellian or bi-Maxwellian, which in turn makes regularization necessary to obtain useful solutions. In previous work velocity-space tomography has been restricted to analysis of $\mathrm{D}_{\alpha}$-light with large Doppler shifts, so-called fast-ion $\mathrm{D}_{\alpha}$-light (FIDA $[65,75]$ ), and to the part of velocity space with energies larger than 15-20 keV. Here we include the thermal feature in the fit and infer the complete velocity distribution function of the deuterium population. The basic plasma parameters $n, v_{d, \|}, T_{\|}, T_{\perp}, p_{\|}$and $p_{\perp}$ can be calculated by taking appropriate zeroth to second moments of the resulting distribution function (section 4). Due to the nonlinear nature of the problem (section 8.1), we calculate the solution iteratively. The minimization problem in iteration step $i$ now becomes

$\operatorname{minimize}\left\|\left(\begin{array}{c}W\left(F_{\text {biMax }}\left(n_{i-1}, v_{d \|, i-1}, T_{\|, i-1}, T_{\perp, i-1}\right)\right) \\ \lambda L\end{array}\right) F_{i}-\left(\begin{array}{c}S \\ 0\end{array}\right)\right\|_{2}$ subject to $F_{i} \geq 0$

where the bi-Maxwellian parameters $n_{i-1}, v_{d \|, i-1}, T_{\|, i-1}$ and $T_{\perp, i-1}$ are found as the lowest moments of $F_{i-1}$ according to section 4 . The results are not sensitive to the start guess. $L$ is a matrix operator effecting a numerical approximation to a gradient [58]. In this is so-called first-order Tikhonov regularization the parameter $\lambda$ balances the requirements to fit the data and smoothness [50].

In figure 7 , the measured total distribution function in discharge \#32323 is compared with the corresponding TRANSP simulation, which is the sum of fast-ion and thermal contributions. The tomography problem was solved for energies to the left of the dashed line. The phase-space densities to the right of the dashed line 
are assumed to be small since no FIDA light above the noise level is observed in the part of the spectra probing only these velocity-space regions [50]. Overall, the measurement and the simulation are in excellent agreement. Figure 8 demonstrates that the simulated and measured loop voltages, the plasma stored energies, and the neutron rates also agree well, indicating that the TRANSP simulation is a good model for this discharge. The observed differences between the tomographic inversion and the simulated ion distribution function in figure 7 partly originate from reconstruction uncertainties and could also partly originate from anomalous effects not caught in the TRANSP simulation.

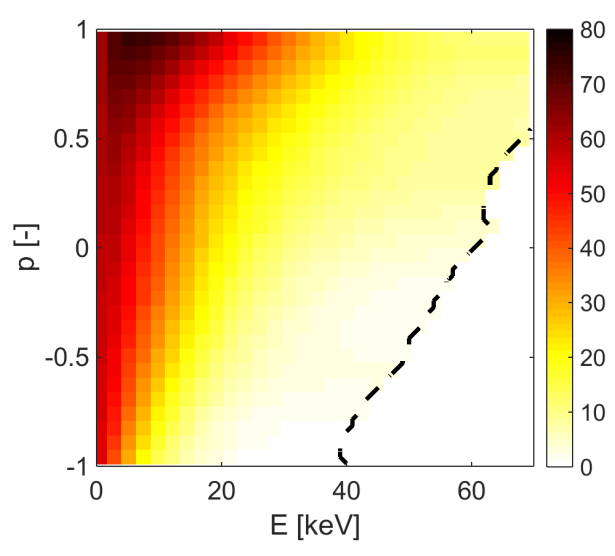

(a) Tomography

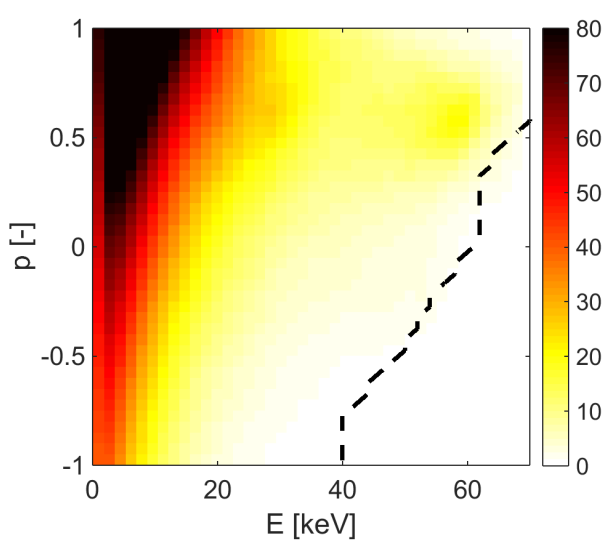

(b) Simulation

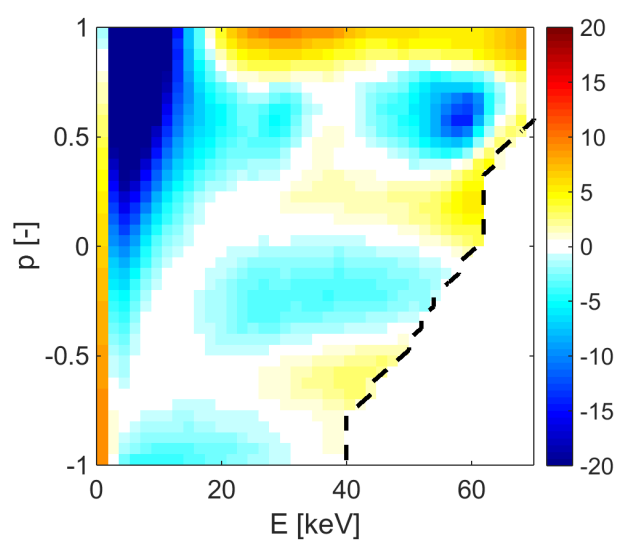

(c) Difference

Figure 7. Comparison of a (a) measurement of $f(E, p)\left[10^{16} /\left(\mathrm{keV} \mathrm{m}^{3}\right)\right]$ by velocityspace tomography and a (b) TRANSP simulation for discharge \#32323 at $1711 \mathrm{~ms}$ before a sawtooth crash in the plasma center. The simulation is the sum of a Maxwellian at $6 \mathrm{keV}$ and the fast-ion velocity distribution function computed with NUBEAM. (c) Difference between (a) and (b).

However, the inference of the low-energy part of velocity space hampers the inference of the high-energy part of velocity space due to the much larger phase-space densities in the low-energy part. For example, the peaks at the NBI injection energies are not found whereas they are routinely found in fast-ion velocity-space tomography 


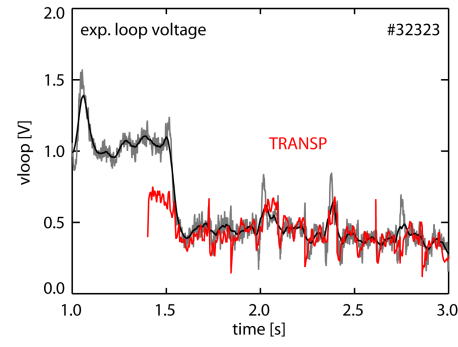

(a) Loop voltage

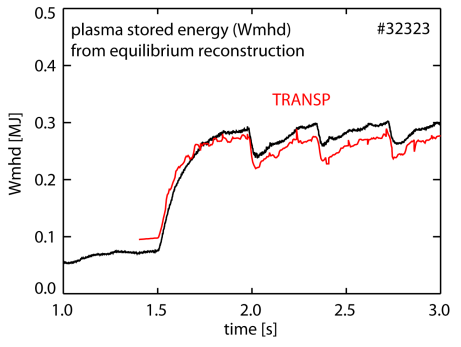

(b) Plasma stored energy

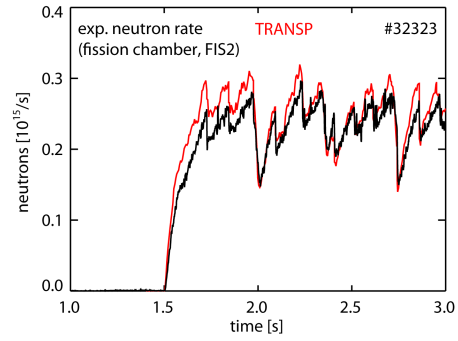

(c) Neutron rate

Figure 8. Comparison of the TRANSP simulation in 32323 and measurements. (a) Loop voltage. (b) Plasma stored energy. (c) Neutron rate.

studies [50]. It will therefore still be advantageous to introduce a lower energy limit in velocity-space tomography studies that are focused on fast ions.

Our goal here is to calculate the lowest moments of the velocity distribution function (section 4). We find that the total density is $2.3 \times 10^{19} \mathrm{~m}^{-3}$ which is comparable to the total density in TRANSP/NUBEAM $\left(2.6 \times 10^{19} \mathrm{~m}^{-3}\right)$. The measured drift velocity is $400 \mathrm{~km} / \mathrm{s}$ and the measured temperatures are $T_{\|}=9 \mathrm{keV}$ and $T_{\perp}=11 \mathrm{keV}$. The corresponding values obtained with TRANSP/NUBEAM when the simulated fast-ion distribution from NUBEAM is accounted for by computing the moments of the total ion distribution function (figure $1(\mathrm{c})$ ) are $T_{\|}=8.3 \mathrm{keV}, T_{\perp}=10.4 \mathrm{keV}, v_{d}=400 \mathrm{~km} / \mathrm{s}$. The agreement between measurements and simulation accounting for fast-ions is excellent. The nominal TRANSP deuterium density $\left(2 \times 10^{19} \mathrm{~m}^{-3}\right)$, temperature $(6 \mathrm{keV})$ and drift velocity $(300 \mathrm{~km} / \mathrm{s})$ are not uniquely measurable quantities. The agreement corroborates our new approach to temperature measurements, and it suggests that the nominal temperatures given by TRANSP need to be regarded as lower bounds. We note that this discharge has a very high $n_{f} / n_{t h}$. In more conventional discharges these differences are smaller according to figure 6 .

As a second example of temperature measurements by velocity-space tomography, we study the impact of electromagnetic wave heating in the ion cyclotron range of frequencies (ICRF). Discharge \#33178 was heated by NBI and ECRH at $5.5 \mathrm{~s}$ and by NBI, ECRH and $2^{\text {nd }}$ harmonic ICRF heating at $7 \mathrm{~s}$ (figure 2). The plasma was at steady-state at both times. The core electron density was $6-7 \times 10^{19} \mathrm{~m}^{-3}$ which is higher than in previous applications of velocity-space tomography. The inversions for both heating schemes are compared in figure 9 . The ICRF heating populates the highenergy space such that the distribution function becomes broader. We find evidence for acceleration above the full NBI energy of $60 \mathrm{keV}$ as expected for the $2^{\text {nd }}$ harmonic ICRF heating scenario which accelerates fast ions from NBI. These results are consistent with recent measurements of the fast-ion velocity distribution function in a comparable plasma scenario [62]. The central boron temperature and the neoclassically computed central deuterium temperature according to TRANSP in the NBI-only phase were about $2.3 \mathrm{keV}$. In the ICRF-heating phase both temperatures are about $1 \mathrm{keV}$ higher according to TRANSP. 
From the inversion, we find deuterium temperatures of $T_{\|}=5 \mathrm{keV}$ and $T_{\perp}=6 \mathrm{keV}$ for the NBI-only phase. The ICRF heating increased both $T_{\|}$and $T_{\perp}$ by $2 \mathrm{keV}$. We find no evidence for preferential heating in the perpendicular direction. The expected tail in the perpendicular direction is likely below the detection limit of FIDA, in particular at high energies exceeding about $150 \mathrm{keV}$. This is in contrast to the strong fast-ion tail in the perpendicular direction observed at JET above $150 \mathrm{keV}[61,76-78]$ as $\gamma$ ray and neutron emission spectroscopy used for the inversion are much more sensitive at very high energies $[57,69,71,72]$. At ASDEX Upgrade such an ICRF heating tail could not be detected by FIDA. Nevertheless, effects of the heating are observed. Fast ions above the critical energy heat preferentially the electrons which in turn quickly approach equilibrium and heat the ions without preferred direction as we observe. At JET, energies below $150 \mathrm{keV}$ were not studied as the $\gamma$-ray measurements have little sensitivity at such energies.

Lastly, we note that a substantial fraction of the ICRF heating accelerates hydrogen [62]. $\mathrm{H}_{\alpha}$-light is therefore also detected by FIDA. According to calculations the hydrogen ions become strongly anisotropic with a long high-energy tail. However, this happens at energies outside the FIDA detection range [62]. As the hydrogen is an impurity species with low concentration, we here neglect the presence of hydrogen.

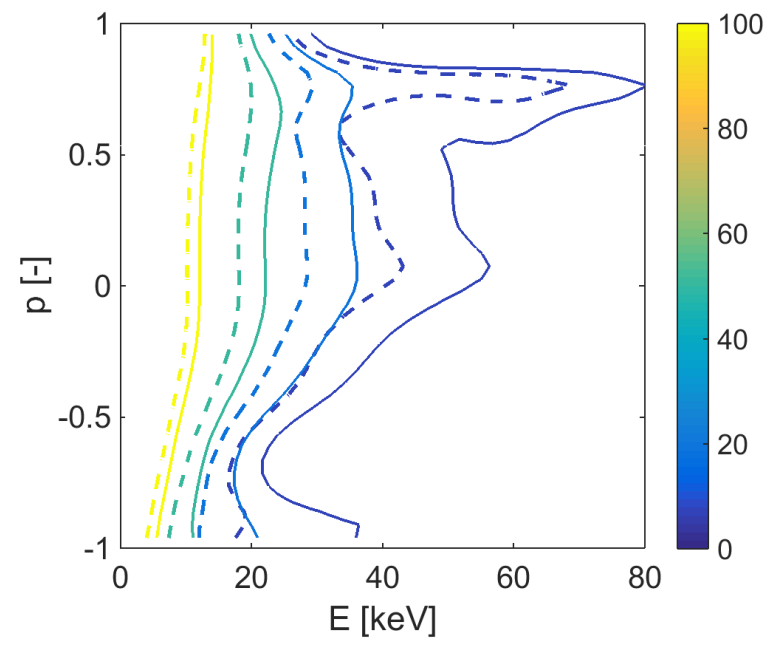

Figure 9. Comparison of measurements of $f(E, p)\left[10^{16} /\left(\mathrm{keV} \mathrm{m}^{3}\right)\right]$ in the plasma center without ICRF heating (dashed lines) and with ICRF heating (full lines) in discharge \#33178.

\section{Conclusions}

We propose two new approaches to measure deuterium densities, drift velocities and parallel and perpendicular temperatures. We further derive an expression for the projection of an arbitrarily drifting bi-Maxwellian distribution function onto the lineof-sight of ion diagnostics, e.g. a CER spectrometer. This projection suggests 
that measurements of any anisotropy are feasible if simultaneous measurements using different lines-of-sight are made. We measured five active spectra of Doppler-shifted $\mathrm{D}_{\alpha}$-light with the five-view FIDA system at ASDEX Upgrade usually used for fast-ion velocity-space tomography.

In our first approach, we fit a bi-Maxwellian to the five simultaneously acquired spectra to obtain the density, the parallel drift velocity and the parallel and perpendicular temperature. Whereas this approach should work well for plasmas that have a bi-Maxwellian distribution function, we demonstrate that it is less reliable in strongly heated, low-density plasmas in fusion devices due to the non-Maxwellian and non-bi-Maxwellian nature of the plasma.

In our second approach, the full velocity distribution function is measured using velocity-space tomography. Here the bulk plasma parameters are obtained as the lowest moments of the measured velocity distribution function. For a Maxwellian distribution these kinetic parameters reduce to the usual Maxwellian parameters. Firstly, we find that the kinetic temperatures are substantially higher than the usual thermal temperatures calculated from impurity temperatures using TRANSP. This is explained by the impact of fast ions. We stress that the kinetic temperatures are experimentally accessible parameters in any plasma whereas the nominal Maxwellian deuterium temperature according to TRANSP is not a uniquely measurable quantity in the presence of an even moderate fast ion population. Secondly, in a plasma heated by NBI at ASDEX Upgrade, we find substantial anisotropy in the plasma. The measured perpendicular temperature is $T_{\perp}=11 \mathrm{keV}$ whereas the parallel temperature is $T_{\|}=$ $9 \mathrm{keV}$. The measured boron temperature is $7 \mathrm{keV}$, and TRANSP determines the single deuterium temperature of $6 \mathrm{keV}$ neoclassically from this boron measurement. However, the parallel and perpendicular temperatures computed as second moments of the sum of the Maxwellian and the fast-ion population from NUBEAM give $T_{\perp}=10.4 \mathrm{keV}$ and $T_{\|}=8.3 \mathrm{keV}$ which are in excellent agreement with the measurement. Similarly, the measured parallel drift velocity of $400 \mathrm{~km} / \mathrm{s}$ is in excellent agreement with TRANSP, if corrected for the impact of fast ions, whereas the nominal deuterium drift velocity computed with TRANSP without any fast-ion correction is $300 \mathrm{~km} / \mathrm{s}$. The measured boron drift velocity in the plasma center is $270 \mathrm{~km} / \mathrm{s}$. As second example, we studied an ICRF heated plasma. Any preferential heating due to ICRF could not be detected using $\mathrm{D}_{\alpha}$-based CER spectroscopy. ICRF elevated the measured parallel and perpendicular temperatures by similar amounts $(2 \mathrm{keV})$.

\section{Acknowledgments}

We thank the ITPA Energetic Particle Physics Topical Group for its support. This work has been carried out within the framework of the EUROfusion Consortium and has received funding from the Euratom research and training programme 2014-2018 under grant agreement No 633053. The views and opinions expressed herein do not necessarily reflect those of the European Commission. 


\section{References}

[1] Meyer H et al 2017 Nucl. Fusion 57102014

[2] Kallenbach A et al 2017 Nucl. Fusion 57102015

[3] Fonck R, Darrow D and Jaehnig K 1984 Phys. Rev. A 29 3288-3309

[4] Isler R C 1994 Plasma Phys. Control. Fusion 36 171-208

[5] Stratton B et al 1991 Nucl. Fusion 31(1) 171-175

[6] Bell R et al 1999 Rev. Sci. Instrum. 70821

[7] von Hellerman M et al 1993 Plasma Phys. Control. Fusion 35 799-824

[8] Bernardo J et al 2016 Rev. Sci. Instrum. 87 11E557

[9] McDermott R et al 2009 Phys. Plasmas 16056103

[10] Pütterich T et al 2012 Nucl. Fusion 52083013

[11] Viezzer E et al 2012 Rev. Sci. Instrum. 83103501

[12] McDermott R M et al 2017 Rev. Sci. Instrum. 88073508

[13] Bortolon A et al 2013 Nucl. Fusion 53023002

[14] Chrystal C et al 2014 Rev. Sci. Instrum. 85 11E302

[15] Chrystal C et al 2016 Rev. Sci. Instrum. $8711 \mathrm{E} 512$

[16] Li Y Y et al 2014 Rev. Sci. Instrum. 85 11E428

[17] Li Y Y et al 2016 Rev. Sci. Instrum. 87 11E501

[18] Field A et al 2009 Plasma Phys. Control. Fusion 51105002

[19] Bell R et al 2010 Phys. Plasmas 17082507

[20] Brau K et al 1983 Nucl. Fusion 23 1643-1655

[21] Jing W et al 2012 Plasma Sci. Tech. 14953

[22] Klyuchnikov L et al 2016 Rev. Sci. Instrum. 87053506

[23] Koide Y et al 2001 Rev. Sci. Instrum. 72119

[24] Berezovskij E et al 1985 Nucl. Fusion 25(10) 1495-1498

[25] Busche E, Euringer H and Jaspers R 1997 Plasma Phys. Control. Fusion 39 1327-1338

[26] Svensson J et al 2001 Plasma Phys. Control. Fusion 43(4) 389-403

[27] Pütterich T, Wolfrum E, Dux R and Maggi C 2009 Phys. Rev. Lett. 102025001

[28] Grierson B A et al 2010 Rev. Sci. Instrum. 81 10D735

[29] Grierson B A et al 2012 Phys. Plasmas 19056107

[30] Grierson B A et al 2012 Rev. Sci. Instrum. 83 10D529

[31] Grierson B A et al 2013 Nucl. Fusion 53063010

[32] Krukin V et al 2013 Plasma Phys. Rep. 39(8) 632-643

[33] Haskey S et al 2016 Rev. Sci. Instrum. 87 11E553

[34] Haskey S et al 2017 JINST 12 C10013

[35] Wurden G et al 1982 Phys. Rev. A 26 2297-2300

[36] Behn R et al 1989 Phys. Rev. Lett. 62 2833-2836

[37] Suvorov E et al 1995 Plasma Phys. Control. Fusion 37 1207-1213

[38] Stejner M et al 2012 Plasma Phys. Control. Fusion 54015008

[39] Stejner M et al 2013 Plasma Phys. Control. Fusion 55085002

[40] Stejner M et al 2015 Plasma Phys. Control. Fusion 57062001

[41] Stejner M et al 2017 Plasma Phys. Control. Fusion 59075009

[42] Nishiura M et al 2014 Nucl. Fusion 54023006

[43] Abramovic I et al 2017 JINST 12 C08015

[44] Abramovic I et al submitted Forward Modelling of Collective Thomson Scattering for Wendelstein 7 - X Plasmas: Electrostatic Approximation

[45] Nocente M et al 2015 Nucl. Fusion 55123009

[46] Pankin A et al 2004 Comp. Phys. Comm. 159 157-184

[47] Stroth U et al 2013 Nucl. Fusion 53104003

[48] Weiland M et al 2016 Plasma Phys. Control. Fusion 58025012 
[49] Jacobsen A S et al 2016 Plasma Phys. Control. Fusion 58045016

[50] Salewski M et al 2016 Nucl. Fusion 56106024

[51] Salewski M et al at press Fusion Sci. Tech. Bayesian integrated data analysis of fast-ion measurements by velocity-space tomography

[52] Salewski M et al 2011 Nucl. Fusion 51083014

[53] Salewski M et al 2012 Nucl. Fusion 52103008

[54] Salewski M et al 2013 Nucl. Fusion 53063019

[55] Salewski M et al 2014 Nucl. Fusion 54023005

[56] Geiger B et al 2015 Nucl. Fusion 55083001

[57] Salewski M et al 2015 Plasma Phys. Control. Fusion 57014021

[58] Jacobsen A S et al 2016 Plasma Phys. Control. Fusion 58042002

[59] Rasmussen J et al 2016 Nucl. Fusion 56112014

[60] Jaulmes F et al 2016 Nucl. Fusion 56112012

[61] Salewski M et al 2017 Nucl. Fusion 57056001

[62] Weiland M et al 2017 Nucl. Fusion 57116058

[63] Geiger B et al 2017 Plasma Phys. Control. Fusion 59115002

[64] Moseev D et al submitted Fast ion diagnostics in magnetically confined plasmas

[65] Heidbrink W W et al 2004 Plasma Phys. Control. Fusion 46 1855-1875

[66] Salewski M et al 2010 Nucl. Fusion 50035012

[67] Salewski M et al 2014 Plasma Phys. Control. Fusion 56105005

[68] Heidbrink W W et al 2007 Plasma Phys. Control. Fusion 49 1457-1475

[69] Jacobsen A S et al 2015 Nucl. Fusion 55053013

[70] Salewski M et al 2015 Nucl. Fusion 55093029

[71] Salewski M et al 2016 Nucl. Fusion 56046009

[72] Jacobsen A S et al 2017 Rev. Sci. Instrum. 88073506

[73] Heidbrink W W et al 2011 Comm. Comp. Phys. 10 716-741

[74] von Hellermann M et al 1995 Plasma Phys. Control. Fusion 37 71-94

[75] Heidbrink W W 2010 Rev. Sci. Instrum. 81 10D727

[76] Litaudon X et al 2017 Nucl. Fusion 57102001

[77] Eriksson J et al 2015 Nucl. Fusion 55123026

[78] Schneider M et al 2016 Nucl. Fusion 56112022 